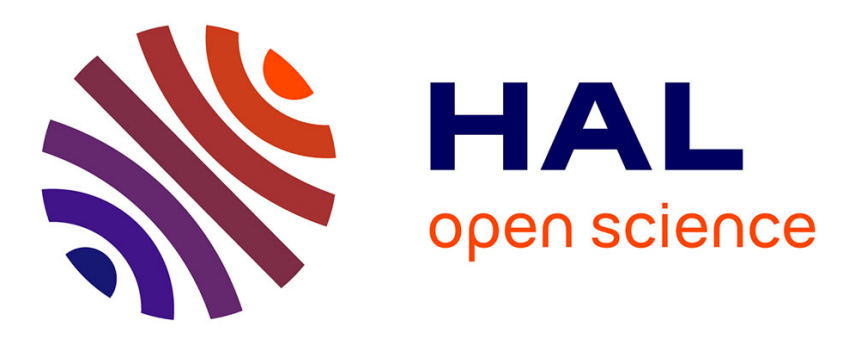

\title{
Présences et absences de la psychanalyse à Cerisy
}

Maïa Fansten

\section{To cite this version:}

Maïa Fansten. Présences et absences de la psychanalyse à Cerisy. Histoire@Politique : revue du Centre d'histoire de Sciences Po, 2013, 20, 10.3917/hp.020.0059 . hal-03478894

\section{HAL Id: hal-03478894 \\ https://cnrs.hal.science/hal-03478894}

Submitted on 19 Jan 2022

HAL is a multi-disciplinary open access archive for the deposit and dissemination of scientific research documents, whether they are published or not. The documents may come from teaching and research institutions in France or abroad, or from public or private research centers.
L'archive ouverte pluridisciplinaire HAL, est destinée au dépôt et à la diffusion de documents scientifiques de niveau recherche, publiés ou non, émanant des établissements d'enseignement et de recherche français ou étrangers, des laboratoires publics ou privés. 
Maïa Fansten, « Présences et absences de la psychanalyse à Cerisy », Histoire@Politique. Politique, culture, société, n² 20, mai-août 2013 [en ligne, www.histoire-politique.fr]

\title{
Présences et absences de la psychanalyse à Cerisy
}

\author{
Maïa Fansten
}

L'après-68 représente pour beaucoup d'observateurs un tournant dans la psychanalyse française. La fin des années 1960 et les années 1970 sont marquées par l'expansion des sociétés psychanalytiques ${ }^{1}$ mais aussi par leur cloisonnement: deux scissions ont lieu dans les années 1960 et le mouvement lacanien se sépare des institutions officielles. Les sociétés de psychanalyse intègrent aussi certains échos de la contestation, en particulier à travers des crises et des innovations en matière de formation. Toutes les sociétés de psychanalyse procèdent alors peu à peu à de profondes réformes de leur système de sélection et de formation au point que l'on peut identifier dans ce domaine un véritable « modèle français ${ }^{2}$ », distinct du modèle traditionnellement adopté à travers le monde (celui de l'International Psychoanalytical Association). Cette période est aussi celle de la formidable expansion du lacanisme, de sa conquête du monde intellectuel français, de son extension politique, de ses affinités avec des problématiques nouvelles, autour par exemple de l'antipsychiatrie ou des travaux féministes. L'expansion du mouvement lacanien va de pair avec une bipolarisation du monde psychanalytique se retranchant en deux camps opposés et hostiles : les freudiens dits " orthodoxes » d'un côté, les lacaniens de l'autre. Quel écho ces événements, ces figures, ces innovations et ces polémiques ont-ils à Cerisy ? Telle est la question qui sera posée ici.

\section{La place de la psychanalyse à Cerisy}

Afin d'y répondre, il convient d'abord de mesurer la place qu'occupe la psychanalyse dans les colloques du centre culturel normand. Le plus logique est de commencer par compter et par caractériser les colloques de psychanalyse qui s'y sont tenus. C'est bien entendu la base de notre analyse et de notre propos. Celle-ci peut être complétée par d'autres indicateurs :

- Le relevé des colloques n'ayant pas nécessairement pour thème la psychanalyse, mais mis en ouvre par des psychanalystes, comme par exemple le colloque de 1968 sur « L'espace du tragique » co-organisé par Anne Clancier et André Green. De tels cas de figure ont été intégrés à l'analyse dans la mesure où ils sont révélateurs du fait

\footnotetext{
${ }^{1}$ Pour en donner la dimension, on peut noter que la Société Psychanalytique de Paris (SPP) passe de 61 membres en 1961 à 439 début 1989. L’École freudienne de Paris, créée par Lacan en 1964, comptait à ses débuts une centaine de membres. En 1980, au moment de sa dissolution, elle atteindra plus de six cents membres.

2 Maïa Fansten, Le divan insoumis. La formation du psychanalyste: enjeux et idéologies, Paris, Hermann, 2006.
} 
Maïa Fansten, « Présences et absences de la psychanalyse à Cerisy », Histoire@Politique. Politique, culture, société, n² 20, mai-août 2013 [en ligne, www.histoire-politique.fr]

que l'approche psychanalytique occupe une place privilégiée dans le traitement du thème choisi et parce qu'on peut supposer qu'un certain nombre de psychanalystes auront été sollicités pour communiquer ou pour participer aux discussions.

- La recherche de la présence de la psychanalyse comme corpus théorique de référence, comme grille de lecture ou d'analyse de façon générale. Nous avons ainsi testé, ponctuellement, un certain nombre de colloques dont l'argument aurait pu justifier que le regard psychanalytique soit convié. C'est le cas, à titre d'exemples, des décades sur la sexualité, sur l'autobiographie, sur le sujet ou encore sur l'androgynie.

- A été également interrogée la place en négatif de la psychanalyse à Cerisy, par ses absences : les colloques qui n'ont pas eu lieu, ou bien les colloques où la psychanalyse n’a pas trouvé la tribune qu'on aurait pu logiquement escompter.

\section{J usqu'en 1974 : une présence discrète et freudienne}

Le premier constat global qui ressort de ces trois approches est que, dans la période allant de 1968 à 1986, le nombre de colloques de psychanalyse reste relativement faible. Si l'on considère l'ensemble des colloques qui se sont tenus à Cerisy entre 1962 et 2007, dix-neuf sont directement liés à la psychanalyse (la psychanalyse est présente dans l'intitulé, elle est donc un objet central de la rencontre).Un seul de ces colloques se tient avant 1968. Pour la période de «l'entre-deux-Mai », on compte quatre colloques et, entre 1986 et 2007, quatorze colloques. Entre 1968 et 1986, la psychanalyse est donc relativement peu présente, avec quatre colloques qui lui sont consacrés. À l'inverse, à partir de la fin des années 1980, le nombre de colloques concernés par la psychanalyse augmente significativement. Sur les dix-neuf colloques recensés sur ce sujet entre 1962 et 2007, la moitié se tient dans les dix dernières années. Cette augmentation doit certes être rapportée à l'augmentation globale du nombre de colloques par an à Cerisy mais pas uniquement. Le contraste est frappant entre la période 1962-1986 et la période 1987-2007. Nous formulerons quelques hypothèses pour comprendre ce contraste. Rémy Rieffel, dans son travail sur Cerisy dans la vie intellectuelle française $\mathrm{du} \mathrm{XX}^{\mathrm{e}}$ siècle à l'occasion du colloque « S.I.E.C.L.E », s'étonnait de l'absence relative de la philosophie, dont il observe qu'elle disparaît des préoccupations principales de Cerisy alors même que «les années soixante et soixante-dix sont propices, en France, à l'éclosion de travaux dans ce domaine et à une contestation véhémente de la philosophie telle qu'elle était enseignée à la Sorbonne». Il concluait que "Cerisy n'a visiblement pas réussi à présenter ce foyer original de la pensée au moment même de la publication de certains ouvrages marquants ${ }^{3} »$. Nous pouvons faire un constat comparable concernant la psychanalyse.

L'entrée de la psychanalyse à Cerisy se fait en 1962, avec le colloque consacré à « L'art et la psychanalyse ». Cette décade est organisée par André Berge, Anne Clancier, Paul Ricoeur et Lothar-Henry Rubinstein. Les communicants psychanalystes - comme un certain nombre de participants - sont des psychanalystes membres de la Société

\footnotetext{
${ }^{3}$ Rémy Rieffel, « Cerisy dans la vie intellectuelle française du XXe siècle », dans François Chaubet, Édith Heurgon, Claire Paulhan (dir.), S.I.E.C.L.E. Colloque de Cerisy. 100 ans de rencontres intellectuelles de Pontigny à Cerisy, Paris, IMEC, coll. « Inventaires », 2005, p. 201.
} 
Maïa Fansten, « Présences et absences de la psychanalyse à Cerisy », Histoire@Politique. Politique, culture, société, n² 20, mai-août 2013 [en ligne, www.histoire-politique.fr]

psychanalytique de Paris (SPP), c'est-à-dire de la société freudienne dite « orthodoxe » car elle est en opposition avec le courant lacanien depuis la scission de 1953. Certains des participants de ce colloque seront d'ailleurs par la suite des habitués de Cerisy, comme Anne Clancier, qui y a organisé de nombreux colloques relatifs à la psychanalyse, mais aussi comme André Green, Nicolas Abraham, J anine Chasseguet-Smirgel, Béla Grunberger, Francis Pasche, Gérard Mendel, etc. Il faut aussi souligner - puisque cela se retrouvera souvent - la présence de psychanalystes étrangers parmi les intervenants de ce colloque: sur les quinzeintervenants psychanalystes, six sont étrangers, surtout anglophones. Ce colloque, qui fait l'objet d'une publication en 1968, représente la psychanalyse appliquée classique, avec des communications sur la littérature classique et la création littéraire contemporaine, la créativité, la musique, les mythes grecs, la sculpture, la peinture, etc. Les références mobilisées sont essentiellement freudiennes.

La psychanalyse revient ensuite, indirectement d'abord, en 1968 avec un colloque sur «L'espace du tragique» organisé par Anne Clancier et André Green. Le terme « psychanalyse » n'apparaît pas dans l'intitulé, mais il s'agit d'un deuxième colloque qui poursuit l'axe d'une psychanalyse appliquée à des thèmes culturels classiques. Le tragique et la tragédie y sont traités par des psychanalyses, mais aussi surtout par des historiens, des critiques littéraires, des philosophes, des poètes. Précision d'importance : ce colloque se tient en juillet 1968. D'après les témoignages, - et bien que « le début fut un peu agité ${ }^{\text {» }}$ selon les termes d'Anne Clancier - , le colloque s'est déroulé dans le calme, contrairement à ce que craignaient ou espéraient certains participants. Ce premier colloque de psychanalyse proche des événements de Mai semble donc peu touché par les remous qui agitent par ailleurs le monde psychanalytique hors les murs.

Il faut dire que certaines des personnalités psychanalytiques habituées de Cerisy sont des membres titulaires de la SPP qui représentent le courant plutôt « conservateur » au sein de l'institution face à la fronde et aux revendications des jeunes générations. C'est en effet autour de 1968 qu'intervient la seconde crise majeure au sein de la SPP, après la scission de 1953. En écho avec les événements extérieurs, un mouvement interne remet en effet en cause l'institution autour des questions de formation et de présélection des candidats. Précisons enfin que ce sont deux habituées de Cerisy, Janine Chasseguet-Smirgel et Béla Grunberger, qui, en 1969, publient sous le pseudonyme d'André Stéphane un ouvrage qui fera grand bruit dans la communauté psychanalytique: cet ouvrage s'intitule L'univers contestationnaire et consiste, comme son titre l'indique, en une attaque en règle de la contestation. C'est dire, donc, que les figures présentes à Cerisy lors de ces premiers colloques ne sont pas celles de la contestation ou de la rupture.

S'il n'apparaît pas, dans les colloques de psychanalyse de Cerisy, d'échos explicites des événements de Mai 68, il n'y pas non plus de traces des événements qui

\footnotetext{
4 Anne Clancier, «Les colloques de psychiatrie, psychanalyse, psychologie», dans ibid., p. 301. Elle précise: «Certains participants annonçaient que Cerisy allait être occupé par des étudiants contestataires, d'autres disaient qu'on ne pouvait plus faire de colloques classiques après les événements de Mai. Finalement, tout se passa fort bien, chacun, et même les plus contestataires, souhaitant faire l'exposé qu'ils avaient préparé. »
} 
Maïa Fansten, « Présences et absences de la psychanalyse à Cerisy », Histoire@Politique. Politique, culture, société, n² 20, mai-août 2013 [en ligne, www.histoire-politique.fr]

marquent la psychanalyse française dans ces années-là. En effet, la scission de 1964 deuxième scission dans l'histoire de la psychanalyse française après celle de 1953 voit la création de l'école de Lacan, l'École freudienne de Paris. Elle entérine le développement du lacanisme en marge de la psychanalyse officielle. Une autre scission en 1969 donne lieu à la création du Quatrième Groupe autour de personnalités issues cette fois de l'école de Lacan, mais en opposition à lui, en particulier à ses propositions relatives à la passe et à son autoritarisme supposé. À partir de 1969, la communauté psychanalytique française est donc scindée en quatre groupes opposés par des conflits plus ou moins violents. Cette configuration durera jusqu'au début des années 1980, puis le paysage sera marqué par le morcellement du mouvement lacanien, divisé en petits groupes après la mort du maître. Les scissions du mouvement psychanalytique français des années 1960 s'accompagnent bien entendu de débats, de textes, de publications, et engagent en particulier une réflexion poussée sur la formation du psychanalyste et sur les institutions psychanalytiques. En même temps qu'une critique de l'institution, ces débats constituent le socle de ce qui deviendra de plus en plus un véritable « modèle français ». Les années qui précèdent sont aussi le moment où se diffusent en France les idées et les pratiques de l'antipsychiatrie. En 1967, Maud Mannoni organise des journées d'études sur les psychoses infantiles où interviendront Laing et Cooper. En 1970, elle publie Psychiatrie et antipsychiatrie. En 1972, paraît L’Anti-Edipe de Deleuze et Guattari. Rien de toutes ces innovations et de ces controverses n'apparaît dans le Cerisy des années 1960.

\section{4 : l'entrée en scène des lacaniens}

Il faut attendre l'année 1974 pour voir l'entrée en scène des lacaniens, avec un colloque qui est resté dans les mémoires, si l'on en juge les témoignages spontanés et les bilans historiographiques existants jusqu'à présent ${ }^{5}$. Malheureusement, ce colloque mémorable n'a pas été publié et nous n'avons donc pas trace de son contenu. Il avait pour thème Le discours psychanalytique et fut organisé par Serge Leclaire. Il a connu un grand succès car on dénombre plus de 110 participants tout en ayant dû refuser du monde. Le thème retenu était très explicitement lacanien puisqu'il faisait écho aux thèses de Lacan sur ce qu'il appelait alors les «quatre discours »: le discours du maître, le discours de l'université, le discours de l'hystérique, le discours de l'analyste. La phase préparatoire de ce colloque avait abouti à la création de cinq groupes de travail : le premier sur la situation de la psychanalyse; le deuxième sur discours psychanalytique et discours politique, le troisième sur discours psychanalytique et discours scientifique; le quatrième sur l'après-coup; et le cinquième sur discours religieux et discours analytique. Parmi les participants, on compta un très grand nombre de lacaniens, dont un certain nombre de psychanalystes belges. On note aussi la présence de nombreux participants anglosaxons, dans le champ de la critique littéraire ou de la philosophie et intéressés par la French Theory (par exemple Barbara J ohnson, Hélène Klibbe, Sidney Levy, Michel

\footnotetext{
5 Voir le site Internet de l'Association des amis de Pontigny Cerisy à la page http://www.cciccerisy.asso.fr/temoignages.html comme celui de Marc Quachebeur, par exemple [lien consulté le 01/ 07/2013].
} 
Maïa Fansten, « Présences et absences de la psychanalyse à Cerisy », Histoire@Politique. Politique, culture, société, n² 20, mai-août 2013 [en ligne, www.histoire-politique.fr]

Pierssens). Étaient également présentes des personnalités comme Catherine Clément, Hélène Cixous, Shoshana Felman, Suzanne Allen. Du côté des freudiens, on retrouve certains habitués de la SPP mais en nombre réduit (seuls participent J acques Clauvel, Jean Cournut, Béla Grunberger et Janine Chasseguet-Smirgel), ainsi que quelques membres des autres groupes (Quatrième Groupe et APF).

Parmi les lacaniens présents, on trouve certaines figures actives et originales de ces années-là. C'est le cas, au premier chef, de Serge Leclaire, qui est aussi à l'origine de la création du département de psychanalyse de l'université de Vincennes et un artisan infatigable, pendant la période, de l'ouverture, du décloisonnement et de la réunification de la psychanalyse française ${ }^{6}$. Sont également présents en 1974 à Cerisy Francis Hofstein et Radmila Zygouris, tous deux à l'origine de la revue L'Ordinaire du psychanalyste, revue qui ne publie que des articles anonymes, créée en 1973. On trouve également Pierre Kahn, qui avait réuni autour de la revue Espaces des personnalités également présentes au colloque de 1974, comme J ean-J acques Blévis ou Alice Cherki. Enfin, la décade accueille aussi J ean Oury, représentant de la psychothérapie institutionnelle depuis 1953 et à l'origine de la fondation de la clinique de La Borde à Cour-Cheverny. À défaut de pouvoir nous référer aux communications, force est de constater que ce colloque réunit un grand nombre de «jeunes» représentants de la génération montante de lacaniens ainsi que des critiques littéraires, des philosophes et des féministes proches de ce courant. Le colloque de Cerisy en 1974 présente donc un échantillon de la variété des idées, des motivations, des initiatives qui émergent ces années-là autour du lacanisme. Selon le témoignage d'Horacio Amigorena, proche de Leclaire, et qui comptait parmi les participants, il y avait de la part de ces derniers la volonté de faire un peu scandale, d'être subversif, de « provoquer l'establishment psychanalytique ». Il se souvient que ce fut un colloque agité, et qu'il faut bien reconnaître qu'avec le recul, l'agitation comptait parfois plus que la valeur théorique de ce qui a été dit?

\section{Après 1977}

En 1977, se tient un colloque consacré à « La psychanalyse des textes littéraires » organisé par Serge Doubrovsky et André J arry. Cette décade renoue donc avec la tradition d'une psychanalyse appliquée. On y retrouve André Green, Anne Clancier, Jean Gilibert mais aussi Alain Costes et Edmundo Gomez-Mango. Pour le reste, les interventions sont surtout le fait de critiques littéraires mobilisant eux-mêmes des outils et des références psychanalytiques. La même année, J acques Schotte et Claude Van Reeth organisent une décade sur « L'analyse du destin de Léopold Szondi ». Ce colloque n'a pas été publié, il n'a pu donc être étudié, mais il est à souligner qu'il s'agit là, non pas de psychanalyse appliquée, mais de l'examen ou de la consécration d'une

\footnotetext{
6 Serge Leclaire, à l'époque de la première scission, a fait partie de la «troïka » œuvrant auprès des instances internationales pour la réintégration de la SFP dans l'IPA. Il est aussi le premier lacanien à rejoindre le groupe Confrontation, qui a réuni des psychanalystes de tous bords de 1973 à 1983, dans un contexte où les conflits entre groupes étaient radicaux et le dialogue inter-institutions quasi nul. Il est également à l'origine de l'Association pour une instance en 1989, qui visait à réunir et à fédérer les psychanalystes français.

${ }^{7}$ Entretien avec Horacio Amigorena, novembre 2007
} 
Maïa Fansten, « Présences et absences de la psychanalyse à Cerisy », Histoire@Politique. Politique, culture, société, n² 20, mai-août 2013 [en ligne, www.histoire-politique.fr]

théorie: la psychologie du destin. En 1980, se tient à nouveau un colloque de psychanalyse appliquée aux arts: il a pour objet «La psychanalyse des arts de l'image » et est organisé par Anne Clancier et Henriette Bessis. Arts plastiques et cinéma y sont étudiés, avec des perspectives psychanalytiques développées par Béatrice Lehalle, Paul Denis, Alain Costes et J ean-Bernard Chapelier, Graziella et Nicos Nicolaïdis, Anne Clancier, J anine Chasseguet-Smirgel. Conférenciers comme participants appartiennent quasiment tous au mouvement freudien : SPP ou APF8 . Après 1986, et en particulier à partir de 1989, on observe une augmentation du nombre et de la fréquence des colloques de psychanalyse. Dans les années 1990, les lacaniens sont de retour, organisant à nouveau des décades. Ce retour se traduit par une répartition plus équilibrée entre freudiens et lacaniens et par un mélange plus fréquent venant marquer la fin des clivages stricts et excluant entre écoles et courants psychanalytiques. Sur une quinzaine de colloques à cette période, quatre seulement sont des colloques de psychanalyse appliquée.

\section{Un bilan}

Pour récapituler, la psychanalyse qui se donne à entendre à Cerisy entre 1968 et 1986 est donc plutôt freudienne. Les lacaniens sont peu représentés jusqu'aux années 1990. Ils arrivent relativement tard, en 1974, et reviennent tard après cela : en 1994, mais surtout en 1996 avec une décade consacrée à Lacan. Cette faible présence du mouvement lacanien est à interroger en regard de son importance dans le contexte intellectuel français de l'époque. Car si l'on en croit Élisabeth Roudinesco « pendant les dix années qui suivent la révolte de mai, le lacanisme, comme forme française de l'aventure freudienne, domine la scène avec une brillance qu'il ne retrouvera jamais ${ }^{9} »$. Or, il est relativement absent de la scène de Cerisy. Un rapide panorama des thèmes de colloques indique que, jusqu'à la fin des années 1980, ce sont les thèmes littéraires ou artistiques qui sont le plus souvent l'objet de l'investigation psychanalytique. Il faut souligner à nouveau que la psychanalyse présente à Cerisy dans ces années-là est une psychanalyse appliquée, une psychanalyse décalée par rapport aux problématiques, aux théories nouvelles, aux confrontations qui agitent et mobilisent le milieu professionnel et intellectuel de cette discipline des années 1960 et 1970.

Il est par exemple étonnant de constater l'absence de la psychanalyse dans au moins deux colloques où elle aurait pu légitimement avoir sa place. D’abord dans le colloque sur la sexualité en 1965, qui réunit des biologistes, des psychologues, ainsi que les psychiatres Marcel Eck et Gaston Ferdière. C'est aussi le cas dans le cadre du colloque intitulé «Penser le sujet aujourd'hui » en 1986, où un seul psychanalyste, Alain Juranville, intervient. On peut être surpris également de l'absence de colloques relayant les travaux des féministes d'inspiration lacanienne, comme Antoinette

\footnotetext{
${ }^{8}$ L'APF est l'Association psychanalytique de France. Produite par l'épisode agité de la scission de 1964, elle est rapidement intégrée à l'IPA. Elle fait donc partie des deux institutions françaises reconnues par l'Association internationale. Pour les distinguer des institutions lacaniennes, il est d'usage de qualifier la SPP et l'APF de « freudiennes » ou d'« orthodoxes ».

${ }_{9}$ Élisabeth Roudinesco, Histoire de la psychanalyse en France, tome 2 : 1925-1985, Paris, Fayard, 1994 [1986], p. 484.
} 
Maïa Fansten, « Présences et absences de la psychanalyse à Cerisy », Histoire@Politique. Politique, culture, société, n² 20, mai-août 2013 [en ligne, www.histoire-politique.fr]

Fouque ou Michèle Montrelay, bien que soient présentes parallèlement à Cerisy des personnalités comme Catherine Clément, Hélène Cixous ou Luce Irigaray. De même, les travaux sur la psychanalyse infantile ou les problématiques relatives à l'éducation, en particulier les travaux de Dolto ne trouvent pas de tribune en Normandie. De manière générale, on constate l'absence des questionnements relatifs à la thérapeutique, à la psychiatrie, alors même que le « champ psy » est traversé par de nombreux débats, innovations et remises en question.

Il conviendrait toutefois de nuancer ce bilan dans la mesure où une étude plus attentive révèlerait que la psychanalyse est néanmoins présente, non pas en tant que discipline ou qu'objet directement débattu mais comme référence, comme grille de lecture, qui vient irriguer les réflexions en philosophie, en littérature, sur les arts, etc. Dans certains cas, cela contribue à l'usage d'une psychanalyse appliquée, mais dans d'autres, ce sont les références freudiennes et lacaniennes sur l'inconscient, le sujet, le langage ou le capitalisme qui sont mobilisées ${ }^{10}$. L'étude des colloques de Cerisy entre 1968 et 1986 depuis l'histoire de la pensée psychanalytique laisse donc apparaître un double mouvement fait de faible visibilité d'un côté et de présence souterraine, de l'autre.

\section{Les raisons d'un rendez-vous manqué}

Une fois que ce constat fait, comment expliquer que l'axe privilégié ait été celui d'une psychanalyse appliquée? À cette question, on peut répondre par trois types d'arguments ou d'hypothèses : un argument pragmatique, un argument stratégique, un argument que l'on pourrait dire «d'adéquation». Le premier argument est simple : l'absence relative de la psychanalyse à Cerisy entre 1968 et 1986 renvoie au fait que les psychanalystes ont d'autres lieux dans lesquels s'organisent des colloques. Comme le dit Anne Clancier, pour les débats théoriques il y a les écoles et les sociétés d'analyse ${ }^{11}$. Et les colloques et rencontres ne manquent pas. Cerisy vient donc compléter une offre déjà très fournie et il est donc logique qu'elle se focalise sur des questionnements moins travaillés dans les cercles analytiques. Le lacanisme, en particulier, est précisément dans ces années-là en train de s'ouvrir à des cercles intellectuels plus larges.

Deuxièmement, le choix de la psychanalyse appliquée, qui plus est aux arts, peut aussi être lu comme une façon de neutraliser le champ, d'éviter les polémiques et les conflits. Nous l'avons vu, la période qui nous intéresse est marquée par le cloisonnement des écoles et des courants de pensée. Freudiens et lacaniens ne dialoguent pas, ou très peu, ils évoluent dans des mondes parallèles, hostiles et ont des représentations schématiques et diabolisées les uns des autres. Sur le plan institutionnel, en tout cas, les choses sont très figées et très conflictuelles. Comme l'écrit Élisabeth Roudinesco : « (...) l'élève admis dans une société, à partir de 1965, n’a plus autant de contacts que celui d'autrefois avec son voisin d'une société rivale. Il

10 On en trouverait des illustrations notamment dans les décades consacrées au Centenaire du Capital (1967), à Roland Barthes (1977) ou bien à « L'autobiographie et l'individualisme en Occident » (1979).

11 Entretien avec Anne Clancier, novembre 2007. 
Maïa Fansten, « Présences et absences de la psychanalyse à Cerisy », Histoire@Politique. Politique, culture, société, n² 20, mai-août 2013 [en ligne, www.histoire-politique.fr]

adhère à une cause, à une doctrine, à un chef ou à un système et se trouve condamné à faire carrière à l'intérieur des murs de son institution ${ }^{12}$. » Ce cloisonnement se retrouve, d'une certaine manière, à Cerisy, comme en témoigne Anne Clancier lorsque, répondant à la question de savoir si le climat était conflictuel entre freudiens et lacaniens à Cerisy, elle répond que non, « ils faisaient leurs colloques de leur côté et nous du nôtre ${ }^{13} »$. L'impératif de ne pas traiter des sujets polémiques entre courants analytiques peut par ailleurs expliquer pourquoi certains colloques n'ont pas eu lieu : il y a eu un colloque sur Freud annulé en 1967; il n'y a pas eu de colloque Lacan avant 1996, soit quinze ans après sa mort ${ }^{14}$. Ce n'est pas un hasard si celui qui fait entrer les lacaniens à Cerisy est Serge Leclaire, qui est précisément un des hommes d'ouverture du lacanisme. En effet, après avoir été à l'origine du département de psychanalyse de Vincennes en 1969, il a été contraint en 1973 par Lacan de démissionner, ce qui entérine ainsi la prise de contrôle du département par Jacques-Alain Miller. On peut donc comprendre que Cerisy ait été à ce moment là particulièrement investi par l'organisateur du colloque de 1974. Enfin, c'est aussi dans ce sens - celui d'une volonté de préserver Cerisy des conflits qui déchirent la communauté psychanalytique - que l'on peut en partie comprendre l'importance des psychanalystes étrangers : ils permettent un dialogue, mais décentré.

Troisièmement, le fait de privilégier à Cerisy une psychanalyse plutôt appliquée peut enfin être interprété comme une prise de position qui est en cohérence avec une certaine tradition et un certain esprit de Cerisy. On pourrait observer que ce n'est pas tant Cerisy qui ne s'adapte pas ou ne s'ouvre pas à « une certaine psychanalyse » - en l'occurrence lacanienne - qu'« une certaine psychanalyse » qui ne s'adapte pas à Cerisy. Autrement dit, la psychanalyse appliquée n'est pas forcément ce qu'il y a de plus «en vogue» dans le milieu psychanalytique de l'époque, mais elle est en revanche tout à fait adaptée à la tradition de Cerisy. Cet axe de questionnement permet, par définition, l'hybridation et le dialogue, qui sont l'originalité revendiquée de Cerisy. Le choix de la psychanalyse appliquée aux arts ou à la littérature permet aussi l'organisation de décades hybrides, mélangeant psychanalystes, écrivains, artistes, philosophes, critiques, etc. Et il est vrai que les colloques plus strictement conçus autour de problématique psychanalytique comportent le risque du cloisonnement et du débat de spécialistes, ce que Cerisy cherche à éviter. En ce sens, Cerisy est tout à la fois un lieu de neutralisation et un lieu de respiration, de déplacement. Mais c'est aussi la tradition de Cerisy que son intérêt privilégié pour le monde littéraire, même si l'intérêt pour la littérature et les arts est plutôt du côté des freudiens que des lacaniens. C'est en tout cas la thèse de Roudinesco qui écrit que :

« (...) Lacan ne s'intéresse ni à la littérature en tant que telle, ni aux écrivains dont il voudrait faire ses adeptes (...). J amais Lacan ne puise dans la littérature le lieu d'une jouissance. Il se saisit d'elle à titre d'ornement. (...) Dans l'entourage du maître on imite cette attitude. Au contraire des psychanalystes de l'autre bord, comme de M’Uzan, Pontalis ou Anzieu, les

\footnotetext{
12 Élisabeth Roudinesco, op. cit., p. 485.

13 Entretien avec Anne Clancier, novembre 2007.

${ }^{14}$ Mais cette date tardive s'explique aussi par les conflits internes au lacanisme à partir de la dissolution del'EFP et de la mort de Lacan.
} 
Maïa Fansten, « Présences et absences de la psychanalyse à Cerisy », Histoire@Politique. Politique, culture, société, n² 20, mai-août 2013 [en ligne, www.histoire-politique.fr]

lacaniens sont incapables de produire sur la littérature autre chose que la confirmation de leurs positions doctrinales ${ }^{15}$. »

Comment, dès lors, rendre compte de la faible présence des lacaniens à Cerisy? Ces derniers ne sont pas venus car ils n'ont pas eux-mêmes investi le lieu. En dehors du colloque de 1974, lorsqu'ils viennent, c'est en suivant une logique de réseaux : via François Wahl, via Tel Quel, via Derrida, via Althusser, etc. Outre l'argument relatif au désintérêt limité porté par ce courant à la psychanalyse appliquée, il faut prendre en compte le fait que le lacanisme, en pleine expansion dans ces années-là, est triomphant sur un certain nombre de scènes et n'a donc d'une certaine manière pas besoin de Cerisy. Le succès considérable de l'EFP dès sa création, celui des Écrits publié en 1966, celui du séminaire de Lacan transféré en 1964 à l’ENS, puis à la faculté de droit au Panthéon en 1969 ; la création de Vincennes et du département de psychanalyse rattaché à l'UER de philosophie : les lieux et les symboles ne manquent pas.

Pourtant, à certains égards, on pourrait considérer Cerisy comme un lieu tout à fait idéal pour ce mouvement en expansion. C'est un lieu «noble», apte à symboliser la reconnaissance et la légitimité du lacanisme. C'est un lieu tiers, ensuite, hors des institutions psychanalytiques officielles mais représentatif du monde intellectuel français. C'est un lieu hybride, enfin, mélangeant philosophes, artistes, universitaires : à l'image du lacanisme en quelque sorte qui, précisément, ouvre la psychanalyse aux sciences humaines, aux philosophes, etc. Aussi faut-il considérer l'hypothèse selon laquelle, c'est Cerisy qui n'a pas investi le lacanisme. En comprendre les raisons profondes exigerait un travail spécifique, mais on peut supposer, entre autres hypothèses, qu'un simple effet «de priorité » a opéré. Les figures plus anciennes de Cerisy étaient avant tout des «freudiens », et certaines d'entre elles étaient très hostiles au lacanisme et inquiètes quant à ses dérives, comme J anine Chasseguet-Smirgel ou André Green, qui a rompu avec Lacan en 1965. Ces personnalités ont peut-être contribué à « isoler » Cerisy du lacanisme, des débats et des controverses du monde psychanalytique. Si Cerisy a été réceptif au lacanisme, c'est indirectement, à travers artistes et philosophes, selon une ligne de diffusion identifiée par exemple par Robert Castel ${ }^{16}$.

Dans son article de2005, Rémy Rieffel, proposait plusieurs modalités de répercussion des colloques de Cerisy : la consécration des grands maîtres à penser

\footnotetext{
15 Élisabeth Roudinesco, op. cit., p. 532.

16 «On sait la séduction que la psychanalyse a tôt exercée sur les membres de l'intelligentsia sophistiquée - en France, par exemple, sur les surréalistes. Mais, indépendamment du jugement que l'on peut porter sur l'œuvre et la pratique de Jacques Lacan, c'est à partir du succès du lacanisme que l'on peut dater la réelle percée de la psychanalyse française hors du ghetto des initiés et des spécialistes. Le séminaire de Lacan se déplace en 1964 à l'Ecole normale supérieure, ce qui montre que son audience a commencé à séduire, depuis quelques années déjà, un noyau représentatif de la haute intelligentsia (Althusser et les althussériens, quelques artistes et philosophes dans le vent). À partir de là, on pourrait suivre une ligne de diffusion intellectuelle, et bientôt mondaine, qui impose la grille d'interprétation psychanalytique lacanienne comme référence privilégiée non seulement pour l'interprétation du psychisme, mais aussi pour celles des productions culturelles, textes littéraires, œeuvres d'art, travail ethnologique: Lacan-Althusser, Lacan-Lévi-Strauss, Lacan-Foucault. » Dans Robert Castel, JeanFrançois Le Cerf, « Le phénomène "psy" et la société française », Le Débat, 3, 1980, p. 23.
} 
Maïa Fansten, « Présences et absences de la psychanalyse à Cerisy », Histoire@Politique. Politique, culture, société, n² 20, mai-août 2013 [en ligne, www.histoire-politique.fr]

(Barthes, Derrida) ; la légitimation des figures subversives (Artaud, Bataille) ; une rampe de lancement pour certains mouvements d'avant-garde (Tel Quel, le Nouveau Roman, la Nouvelle Critique); un déclencheur de problématiques nouvelles (épistémologie, sciences cognitives). Si on se réfère à cette grille de lecture, force est de constater que les colloques de psychanalyse à Cerisy, bien qu'ayant pour certains connu de grands succès, n'ont véritablement permis aucune de ces répercussions. Dans la période de «l'entre-deux-Mai », Cerisy a privilégié les grandes figures freudiennes, comme André Green, et une psychanalyse de qualité, mais déconnectée des problématiques contemporaines, de l'avant-garde ou de la mise en cause. Cerisy est en particulier resté hermétique au mouvement lacanien. Il n'a pas reflété les aspects les plus novateurs et subversifs des années 1960 et 1970 et s'est fait seulement l'écho parfois lointain et souterrain de démarches et de lectures lacaniennes, souvent autour de Barthes, Derrida ou Althusser, sans que le lacanisme soit jamais au centre.

L'histoire de la psychanalyse française, et plus particulièrement celle du lacanisme, bien qu'imbriquée dans celle de l'après-68, a ses propres temporalités : la mort de Lacan en 1981 va bien sûr modifier le paysage et produire des effets pendant une bonne partie de la décennie 1980. Après la dissolution de l’EFP et la mort du maître, c'est une période de crise et d'éclatement qui s'engage. Il faudra quelques années avant que s'apaisent les tensions nées à ce moment. Et certaines de ces ruptures sont d'ailleurs toujours agissantes. C'est seulement à la fin des années 1980 que l'on constate les premiers signes ou les premières tentatives de réconciliation. Dans la communauté analytique dans son ensemble, avec l'Instance Ordinale en 1989 et les États Généraux de la psychanalyse en 2000 ; dans la communauté lacanienne avec l'Interassociatif des psychanalystes et Convergencia au début des années 1990. À Cerisy, les séminaires « Depuis Lacan » en 1996 et « 2001, Lacan dans le siècle » s'inscrivent aussi dans ce contexte.

Ajoutons également qu'à partir des années 1990, la psychanalyse connaît un certain recul : elle perd en visibilité, en influence, elle ne règne plus sur la psychiatrie, elle est en perte de vitesse à l'Université, elle est concurrencée par d'autres paradigmes et par d'autres pratiques. Bref, ses colloques n'attirent plus les foules. Le contraste que nous avons observé entre les deux périodes - 1968-1986 et 1986-2007- est aussi le reflet de ces profonds changements. Le champ psychanalytique s'est quelque peu réunifié, il s'est en tout cas ouvert au dialogue en interne. Les décades proprement psychanalytiques sont moins problématiques. Le temps des bilans et des hommages réunit davantage. La psychanalyse est aussi moins conquérante, elle n'est plus ce foyer de «colonisation de la culture intellectuelle ${ }^{17}$ », on vient moins à elle. On comprend l'avantage qu'elle ait eu alors à se montrer hors les murs, à dialoguer avec l'extérieur. Et donc à réinvestir Cerisy.

\section{L'auteur}

Maïa Fansten est sociologue, maîtresse de conférences à l'université Paris Descartes et membre du Cermes 3 (Centre de recherche médecine, sciences, santé, santé mentale).

\footnotetext{
${ }^{17}$ Selon l'expression de Sherry Turkle, La France freudienne, Paris, Grasset, 1982, p. 25.
} 
Maïa Fansten, « Présences et absences de la psychanalyse à Cerisy », Histoire@Politique. Politique, culture, société, n² 20, mai-août 2013 [en ligne, www.histoire-politique.fr]

Ses travaux portent sur la sociologie de la psychanalyse, des pratiques, des savoirs et des catégorisations en santé mentale. Elle est l'auteure de Le divan insoumis paru aux éditions Hermann en 2006.

\section{Résumé}

La période des années 1960 aux années 1980 correspond en France à une période d'innovations, de réformes et d'expansion de la psychanalyse, en particulier à travers l'influence croissante du lacanisme, mais aussi des controverses et des scissions qu'il provoque au sein du mouvement psychanalytique français et des spécificités qu'il lui imprime. Quel écho ces événements, ces figures, ces innovations et ces polémiques ont-ils à Cerisy ? Étonnement, la psychanalyse y est relativement peu présente, alors même qu'il s'agit d'une période de bouillonnement et de forte visibilité dans le champ intellectuel et culturel français. La psychanalyse qui est représentée dans ces années à Cerisy est quelque peu en décalage avec la réalité des débats et des apports qui nourrissent la discipline: les colloques mettent en scène de façon privilégiée une psychanalyse freudienne orthodoxe (les lacaniens sont très peu représentés) et une psychanalyse souvent appliquée (aux thèmes littéraires et artistiques). Aussi la psychanalyse est-elle à la fois peu visible et présente de façon indirecte et souterraine à l'occasion de colloques qui ne la prennent pas directement ou explicitement pour objet ou pour référence. Cet article propose d'examiner et d'analyser cette singulière présence / absence de la psychanalyse à Cerisy.

Mots clés : Cerisy ; psychanalyse ; lacanisme ; sociologie ; savoirs.

\section{Abstract}

The 1960s, 1970s and 1980s are pivotal decades of innovation, reform and expansion of psychoanalysis in France, characterized by the growing influence of Lacan and Lacanism, and the numerous controversies and splits it caused within the French psychoanalytic movement. What are the repercussions of these events, innovations and controversies at Cerisy? Surprisingly, while it's a period of ferment and high visibility of psychoanalysis in the French intellectual and cultural fields, psychoanalysis is not very represented at Cerisy during this period. Only a few symposia are dedicated to psychoanalytical matters and when they are, the subjects chosen to be discussed are somewhat removed from the reality of innovating or controversial psychoanalysis : mainly freudian orthodox stream (Lacanian are poorly represented) and often applied psychoanalysis (to literary and artistic matters). This paper aims to examine and analyze this singular presence/ absence of psychoanalysis at Cerisy.

Key words : Cerisy; Psychoanalysis; Lacan; Sociology; Knowledge.

Pour citer cet article : Maïa Fansten, « Présences et absences de la psychanalyse à Cerisy », Histoire@Politique. Politique, culture, société, n² 20, mai-août 2013 [en ligne, www.histoire-politique.fr] 